2021, Band 9, Heft 1/2

Seiten 201-204

zeitschrift-suburban.de

10.36900/suburban.v9i1/2.682

\title{
Kein Ende, sondern eine Veränderung des Städtischen
}

Kommentar zu Stefan Höhne und Boris Michel „Das Ende des Städtischen?

Pandemie, Digitalisierung und planetarische Enturbanisierung“

Stefan Höhne, Boris Michel ,Das Ende des Städtischen? Pandemie, Digitalisierung und planetarische Enturbanisierung'

Kommentare von: Marcelo Lopes de Souza, Matthias Naumann, Anke Strüver, Markus Kip, Hannah Schilling, Roger Keil, Christian Haid, Anna-Lisa Müller, Nikolai Roskamm

Höhne und Michel (2021) greifen in ihrem Beitrag ein aktuell dominierendes Thema auf: die Covid-19-Pandemie. Medial, politisch, aber auch im Alltag gibt es kaum Momente, in denen dieses Thema derzeit (Januar 2021) nicht präsent ist. Die Autoren diskutieren die spezifischen Auswirkungen der Pandemie sowie der durch sie vorangetriebenen Digitalisierungsprozesse auf Städte.

Höhne und Michel skizzieren „neun Symptome einer Krise der Städte“ (2021: 142) und prognostizieren ein „Ende des Städtischen“. Die Krise der Städte machen sie an zentralen, traditionell in der Stadtforschung verwendeten Merkmalen des Städtischen fest: Dichte, Funktionstrennung und spezifisch urbane Gesellschaftsstrukturen, die etwa durch Individualisierung und soziale Interaktionen mit Fremden gekennzeichnet sind. Die Pandemie und die Maßnahmen zu ihrer Eindämmung führten, so die Autoren, gerade zum Verlust dieser spezifisch urbanen Merkmale, etwa indem Begegnungen mit Fremden sowie die zwanglose Nutzung des öffentlichen Raums problematisiert werden, oder aber durch die Aufhebung der Funktionstrennung von Arbeits- und Lebensorten durch die Einführung eines Primats des Homeoffice. Perspektivisch könne am Ende einer solchen Entwicklung „eine tiefgreifende Transformation dessen, was man gemeinhin als urbane Lebensweise und Kultur bezeichnet“ (ebd.: 146), stehen.

In meinem Kommentar möchte ich auf die Symptomanalyse und These von Höhne und Michel mit zwei Gegenbeobachtungen und -thesen antworten: (1) Wir haben es mit einer Neuformierung sozialer Interaktionen zu tun, die maßgeblich von Digitalisierung geprägt ist; (2) wir beobachten eine Restrukturierung des öffentlichen Raums und dessen, was als Öffentlichkeit konzipiert wird. Dabei ist die Stadt ein Ort - wenn auch nicht der einzige -, auf den sich diese Transformationen auswirken und an dem sie zu beobachten sind.

Ich stimme den Autoren also zu, dass die aktuelle Covid-19-Pandemie zu fundamentalen Transformationen des Urbanen führen kann, stelle aber zur Diskussion, dass wir es nicht mit einem Verschwinden, sondern mit einer Veränderung des Städtischen zu tun haben. Die Thesen, die ich formuliere, fußen auf punktuellen alltagsweltlichen Beobachtungen einer Stadtforscherin 
- mir selbst - sowie systematischen Beobachtungen der Restrukturierungen des öffentlichen Raums und ersten Analysen derselben (siehe auch Tuitjer/ Tuitjer/Müller 2020).

\section{Neuformierung sozialer Interaktionen}

Die Pandemie hat die sozialen Interaktionen vor Ort maßgeblich verändert. Die Autoren beschreiben sehr richtig, wie der „Kontakt zu Fremden“(Symptom 6) (Höhne/Michel 2021: 144) derzeit problematisiert und pathologisiert wird. Hinzu kommt, dass die Performativität der körperlichen Interaktion, also die zutiefst körperlichen und verkörperlichten Umgangsweisen miteinander, ersetzt werden (sollen) durch räumlich distanzierte Modi der Interaktion. Der „Namaste“-Gruß, den Österreichs Bundespräsident Alexander van der Bellen vorschlug (Wiener Zeitung Online 2020), wurde ebenso eingeführt wie der Ellenbogen- oder Fuß-Check als Ersatz für den Handschlag oder die Umarmung. Auch das Andeuten von Umarmungsgesten bei gleichzeitigem Einhalten der geforderten Distanz von 1,50 Metern lässt sich beobachten.

Damit formieren sich neue körperliche Interaktionsformen. Darüber hinaus sind digitalisierte Interaktionen zu finden, die an die Stelle von analogen Interaktionen treten: die gemeinsame digitale Mittagspause in einem virtuellen Raum oder der kollegiale Spaziergang an der frischen Luft anstelle des kollegialen Kaffees, bei dem berufliche, aber auch private Angelegenheiten besprochen werden. Hier können wir weniger einen Ersatz als vielmehr eine Ergänzung der erlernten, etablierten, verkörperlichten Interaktionsformen finden. War es die körperliche und räumliche Nähe, die von emotionaler Intimität zeugte, so tritt nun das Doppel von digitaler Nähe und räumlicher Distanz an ihre Stelle.

Anders als von den Autoren diagnostiziert (Symptom 8), lässt sich hier in vielen Fällen gerade keine neue „Form von Vereinzelung“ (Höhne/ Michel 2021: 145) feststellen, sondern vielmehr die Veränderung zutiefst sozialer, selbst gewählter und damit auch - der Argumentation der Autoren folgend - urbaner Interaktionen. So lässt sich mit der städtischen digitalen Kommunikation die Geschichte der engen Wechselbeziehung von Medien und Stadt fortschreiben (exemplarisch Hepp et al. 2018; für soziale Medien siehe auch Evans/Saker 2017).

Interaktionen sind auch in ihrer digitalen Form von urbanen Merkmalen geprägt: In der Stadt sind erstens die infrastrukturellen Bedingungen für die Nutzung digitaler Dienste im Durchschnitt qualitativ besser als in ruralen oder peripheren Gegenden. Digitale Infrastrukturen unterscheiden sich damit untereinander auch hinsichtlich ihrer räumlichen Verortung, ebenso wie dies für analoge Infrastrukturen (Verkehr, Dienstleistungen oder Ähnliches) gilt. Zweitens lassen sich die Angebote der digitalen Interaktion in urbanen Kontexten eher in vollem Umfang nutzen als in ländlichen Räumen. So können sich etwa in sozialen Medien mehr vor Ort ansässige Akteure zu einem lokalen Thema austauschen; Essenslieferdienste sind in anderer Quantität und Qualität vorhanden und können genutzt werden, um das urbane ,Ausgeherlebnis“ in den privaten Raum hineinzuholen. Das Merkmal der Dichte, das schon Wirth für die Stadt als charakteristisch identifizierte, hinterlässt seine Spuren also auch in der digitalen Welt (Wirth 1938). 


\section{Restrukturierung von öffentlichem Raum und Öffentlichkeit}

Der öffentliche Raum hat sich seit März 2020 in Deutschland deutlich in seiner Funktion und seiner Gestalt verändert. Ich stimme den Autoren zu, wenn sie konstatieren, dass die urbane Dichte in dieser pandemischen Zeit „als Gefahr“ (Symptom 1) (Höhne/Michel 2021: 142) gerahmt wird. Allerdings würde ich auch hier weniger die „Zusammenbrüche urbaner Kultur" (Symptom 5) (ebd.: 144) feststellen wollen als eher eine temporäre Funktionsveränderung des (städtischen) öffentlichen Raums: weg von verorteten Konsum- und Festivalisierungspraktiken hin zu alternativen Freizeitpraktiken. Sportliche Betätigung im Freien war selten eine so stark praktizierte Tätigkeit wie in den Monaten, in denen Turnhallen und Fitnessstudios geschlossen waren. Outdoor-Sportkurse wurden im Sommer in Parks abgehalten, seit Jahren ungenutzte Fahrräder reaktiviert. Nicht zuletzt an den leer gekauften Fahrradläden ist zu erkennen, welche neuen Praktiken der Nutzung des öffentlichen Raums sich hier aufgetan haben.

Zusätzlich wird, so mein Argument, der analoge öffentliche Raum um einen digitalen öffentlichen Raum ergänzt, etwa wenn die Royal Albert Hall Livekonzerte im Livestream und ohne monetäre Zugangsbeschränkung zeigt (zum Beispiel Royal Albert Hall 2020) oder Parlamentssitzungen digital übertragen werden. Zudem haben sich Apps wie Coffitivity[1] und Playlists von Streamingdiensten etabliert, die Hintergrundgeräusche von Bars oder Restaurants anbieten. So kann ein Gefühl des Ausgehens und Teilhabens am urbanen (Nacht-)Leben in die Privatheit des Zuhauses hineingeholt werden.

Dass dies mit neuen oder anderen Formen von Barrieren einhergeht, wird dabei auch und zu recht diskutiert: Technologien wie Computer oder Smartphones, Headsets und Lautsprecher, aber auch finanzielle Ressourcen und digitale Kompetenzen für einen Internet- oder Mobilfunkzugang spielen in digitalisierten öffentlichen Räumen die Rolle von Türsteher_innen, Ticketverkaufsstellen oder physischen Türen, die geöffnet werden müssen.

Um die Frage zu beantworten, inwiefern es zu einem „Ende des Städtischen" kommen kann, wie von Höhne und Michel zur Diskussion gestellt, braucht es umfassende empirische Untersuchungen. Außerdem ist es wichtig, die Vielfältigkeit der Städte - global, aber auch national und regional - zu berücksichtigen. Historische Entwicklungen spielen ebenso eine Rolle wie die politischen Kontexte und die kollektiven Erfahrungen der jeweiligen Stadtgesellschaft. Eine (erste) Antwort auf diese wichtige Frage kann zum jetzigen Zeitpunkt aus meiner Sicht immer nur eine lokale sein. Inwiefern die Pandemie tatsächlich über Jahrzehnte gelernte, inkorporierte Praktiken der Interaktion und der Nutzung der Städte und ihrer öffentlichen Räume so verändern kann, dass das, was wir qualitativ als „Stadt“, „städtisches Leben“ und „urbane Kultur“ beschreiben, nicht mehr zu beobachten ist, wird auch von der Dauer der Pandemie und der mit ihr einhergehenden politischen, medizinischen und rechtlichen Auflagen abhängen. Derzeit sehe ich eine temporäre Veränderung von Alltagsroutinen, die ausreichend internalisiert sind, um den Alltag zu verändern, aber noch nicht inkorporiert wurden wie könnte man sonst beim Verlassen des Hauses doch immer wieder seine Maske vergessen? 
So haben wir es also aus meiner Sicht mit einer Neuformierung sozialer Interaktionen zu tun, die deutlich von Digitalisierungsprozessen geprägt ist. Diese neuen Formen der Interaktion ebenso wie die Digitalisierung bewirken außerdem eine Veränderung des öffentlichen Raums und unseres Verständnisses von Öffentlichkeit. In der Stadt zeigen sich diese neuen Formen deutlich; die Rekonfigurationen sind allerdings nicht auf diese beschränkt, sondern zeigen sich andernorts in ähnlicher Weise. Die je charakteristischen räumlichen Bedingungen vor Ort bewirken dabei eine je spezifische Ausgestaltung dieser Neuformierungen.

\section{Endnoten}

[1] Vgl. https://coffitivity.com.

\section{Autor_innen}

Anna-Lisa Müller, Humangeographin und Soziologin, arbeitet zu Stadtentwicklung und Migration mit Schwerpunkt auf dem Nexus von Architektur und Sozialem sowie von Materialität und Ortsbindungen.

anna-lisa.mueller@uni-osnabrueck.de

\section{Literatur}

Evans, Leighton / Saker, Michael (2017): Location-based social media. New York: Palgrave Macmillan.

Hepp, Andreas / Kubitschko, Sebastian / Marszolek, Inge (Hg.) (2018): Die mediatisierte Stadt. Kommunikative Figurationen des urbanen Zusammenlebens. Medien-Kultur-Kommunikation. Wiesbaden: Springer VS.

Höhne, Stefan / Michel, Boris (2021): Das Ende des Städtischen? Pandemie, Digitalisierung und planetarische Enturbanisierung. In: sub \urban. zeitschrift für kritische stadtforschung 9/1-2, 141-149.

Royal Albert Hall (2020): Royal Albert Home. Sophie Hunger. https://www.royalalberthall. com/tickets/events/2020/royal-albert-home-sophie-hunger/ (letzter Zugriff am 26.1.2021).

Tuitjer, Leonie / Tuitjer, Gesine / Müller, Anna-Lisa (2020): COVID-19 und das (neu) Lernen von sozialen Infrastrukturen. Ergebnisse aus einem (auto-)ethnographischen Projekt in Krisenzeiten. Paper präsentiert beim Online-Symposium: COVID-19 als Zäsur? Geographische Perspektiven auf Räume, Gesellschaften und Technologien in der Pandemie. Heidelberg/Innsbruck: Universität Heidelberg/Universität Innsbruck.

Wiener Zeitung Online (2020): „Die Rede des Bundespräsidenten Van der Bellen im Wortlaut“. In: Wiener Zeitung Online, vom 14.3.2020. https://www.wienerzeitung.at/ nachrichten/politik/oesterreich/2054366-Die-Rede-des-Bundespraesidenten-Van-derBellen-im-Wortlaut.html (letzter Zugriff am 23.11.2018).

Wirth, Louis (1938): Urbanism as a way of life. In: The American Journal of Sociology 44/1, $1-24$. 